\title{
A Reproducible Protocol to Measure the Critical Swimming Speed of Adult Zebrafish
}

Yuma Wakamatsu, Makoto Kashima and Hiromi Hirata*

Department of Chemistry and Biological Science, College of Science and Engineering, Aoyama Gakuin University, Sagamihara 252-5258, Japan

*For correspondence: hihirata@chem.aoyama.ac.jp

[Abstract] The quantitative measurement of water flow-induced swimming of fish species using a swimmill is a powerful method to evaluate motor ability of individual fish. Zebrafish is a commonly used vertebrate that enables the study of morphological, physiological and behavioral characteristics associated with genes. We here established a reproducible method that allows to measure the body length and the critical swimming speed of adult zebrafish using a swimmill.

Keywords: Motor, Speed, Swimmill, Swimming, Zebrafish

[Background] To evaluate motor ability of fish, swimmill systems have been used for measurement of critical swimming speed $\left(U_{\text {crit }}\right)$, which is the maximum water velocity in which fish can keep swimming. Many different swimmill equipments and protocols have been used for zebrafish studies (Table 1) and thus, it was difficult to compare results of different experiments and different protocols due to the lack of detailed calibration methods and protocols. We applied commercially available swimmill equipment Swim Tunnel Respirometer, which is the major and standard one among zebrafish researchers, established a reproducible protocol for obtaining basic swimming characteristics of adult zebrafish, such as adequate water temperature and adequate length of caudal fin among various wild-type strains (Wakamatsu et al., 2019). We here describe the detailed methods to monitor dissolved oxygen for the estimation of oxygen consumption in swimming, to measure body size of adult zebrafish, to conduct calibration of water velocity, to acclimate zebrafish to the narrow swimming chamber and to measure swimming speed. This protocol would be useful for researchers who use swimmill. 
Please cite this article as: Wakamatsu et. al., (2020). A Reproducible Protocol to Measure the Critical Swimming Speed of Adult Zebrafish,Bio-protocol 10 (16): e3712. DOI: 10.21769/BioProtoc.3712.

Table 1. Swimmill equipments. The absolute value of $U_{\text {crit }}$ measured in adult wild-type zebrafish (3-12 months old) appears to vary by the difference of the swimmill equipments. This is probably due to the size difference of swimming chamber that generates uneven water flow between center and peripheral. Therefore, the comparison of $U_{\text {crit }}$ should be done in the assays that use the same equipment.

\begin{tabular}{|c|c|c|}
\hline Swimmill & $U_{\text {crit }}(\mathrm{cm} / \mathrm{s})$ & References \\
\hline & \multirow{5}{*}{$25-40$} & Massé et al., 2013 \\
\hline Swim Tunnel Respirometer model 170 ml & & Thomas et al., 2013 \\
\hline $230 \mathrm{~V}, 50 \mathrm{~Hz}$ & & Lucas et al., 2016 \\
\hline \multirow[t]{2}{*}{ Loligo Systems \#SW10000 } & & Parisi et al., 2018 \\
\hline & & Wakamatsu et al., 2019 \\
\hline Swim Tunnel Respirometer model $5 \mathrm{~L}$ & \multirow{3}{*}{$70-90$} & \\
\hline $230 \mathrm{~V}, 50 \mathrm{~Hz}$ & & 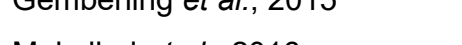 \\
\hline Loligo Systems \#SW10050 & & Mokalled et al., 2016 \\
\hline \multirow{4}{*}{$\begin{array}{l}\text { Swim Tunnel Respirometer model } 10 \mathrm{~L} \\
230 \mathrm{~V}, 50 \mathrm{~Hz}\end{array}$} & \multirow{4}{*}{$40-80$} & Gilbert et al., 2014 \\
\hline & & Conradsen and McGuigan, 2015 \\
\hline & & Conradsen et al., 2016 \\
\hline & & Messerli et al., 2020 \\
\hline Swimmill system made by the author & $50-65$ & Plaut, 2000 \\
\hline Swimmill system made by the authors & 55 & Palstra et al., 2010 \\
\hline Swimmill system made by the authors & $25-30$ & Leris et al., 2013 \\
\hline Flume & $35-45$ & Widrick et al., 2018 \\
\hline
\end{tabular}

\section{Materials and Reagents}

1. $50 \mathrm{ml}$ conical tube (TrueLine, catalog number: TR2005)

2. Zebrafish wild-type strain 3-9 months old adult (Neos Pet Shop, catalog number: a2-1020v3)

3. Green fluorescent PE micro spheres (Loligo Systems, catalog number: AC10555)

4. Oxygen can $5 \mathrm{~L}$ (Pinole, catalog number: 4530896201169)

5. Sodium sulfite (Wako, catalog number: 192-03415)

\section{Equipment}

1. Swim Tunnel Respirometer model 170 ml, 230 V, $50 \mathrm{~Hz}$ (Loligo Systems, model: SW10000)

2. High-speed video camera (Imaging Development Systems, model: UI-3240CP-C-GL)

3. Green laser pointer $300 \mathrm{~mW}, 532 \mathrm{~nm}$ (Loligo Systems, model:AC10551)

4. Eheim pump $5 \mathrm{~L} / \mathrm{min}, 230 \mathrm{~V}, 50 \mathrm{~Hz}$ (Loligo Systems, model: PU10050)

5. Aquarium heater $300 \mathrm{~W}$ (Nisso, model: R300W)

6. $10 \mathrm{ml}$ measurement cylinder (Iwaki, model:3022CYL10S)

7. Electric balance $0.01 \mathrm{~g}$ resolution (A\&D, model: FZ-3000i) 


\section{Software}

1. AutoResp ${ }^{\mathrm{TM}}$ version 2 (Loligo Systems \#AR12600)

2. uEye Cockpit (Imaging Development Systems \#UI-3240CP-C-GL)

3. Digital Particle Tracking Velocimetry (DPTV) (Loligo Systems \#AC10550)

4. ImageJ (https://imagej.nih.gov/ij/)

5. Microsoft Excel (Microsoft)

\section{Procedure}

A. Calibration of water velocity

This system consists of an outside tank and a double cylinder (Figure 1A). The outside tank contributes to maintain water temperature of the double cylinder, which is composed of an external cylinder and an inner swimming chamber (Figure 1B). A propeller in the swimming chamber generates water flow in the double cylinder by controlling the propeller rotation depending on an input voltage controlled by the AutoResp software. It is necessary to calibrate the input voltage and water velocity. This calibration can be done by flowing green fluorescent micro beads in the swimming chamber using high-speed camera controlling software uEye Cockpit and analyzing velocity of moving beads using DPTV. 


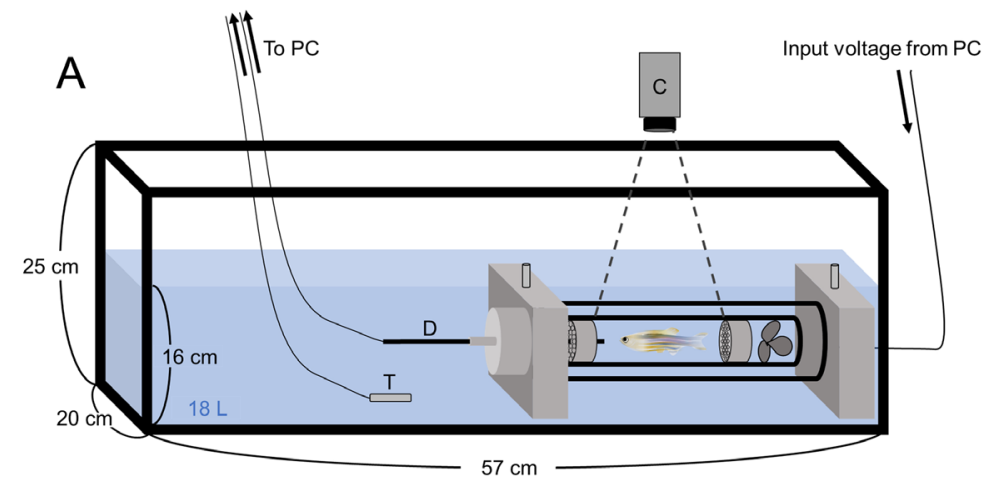

B
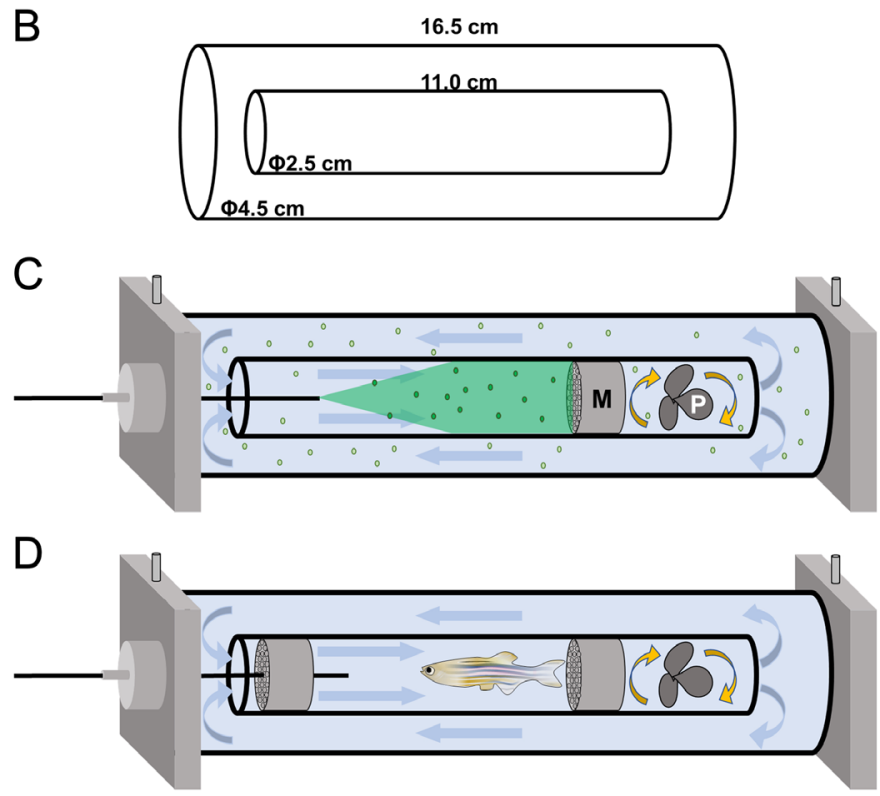

Figure 1. Structure of the swimmill. A. The schematic diagram of the Swim Tunnel Respirometer. C: high speed video camera; D: dissolved oxygen densitometer, T: thermometer. B. Size of the double cylinder. C. An enlarged view of the double cylinder. The propeller rotation generates a directional water flow in the double cylinder. Fluorescent beads flow in the double cylinder and glitter by receiving the excitation light from the green laser pointer. M: mesh; P: propeller. D. In the presence of water flow, zebrafish keep swimming by the same speed with water velocity to stay at the same position. Two plastic meshes were put in the swimming chamber to avoid the fatigued fish hit the propeller.

1. Set up the Swim Tunnel Respirometer with $18 \mathrm{~L}$ of water in the outside tank and inside double cylinder along with a heater that maintain the outside water temperature at $26 \pm 0.2^{\circ} \mathrm{C}$ (Video 1). Fish is not necessary for the calibration of water velocity. 


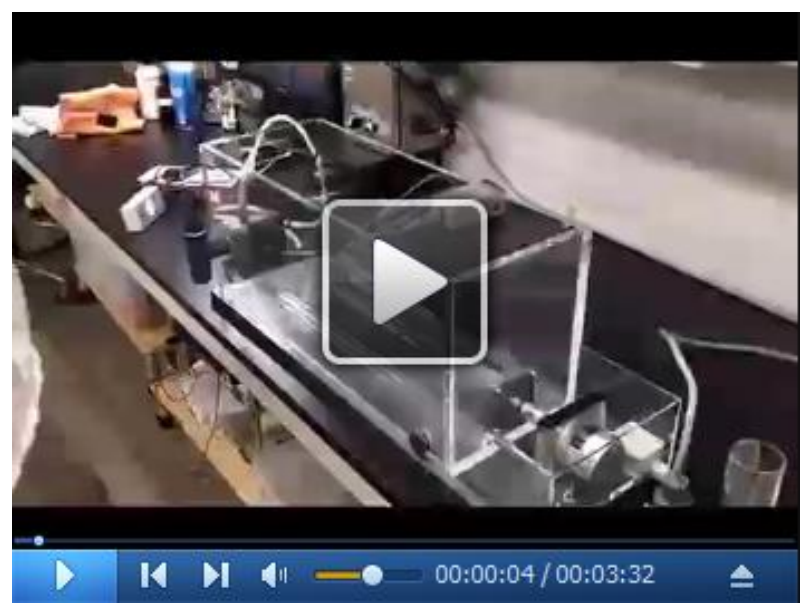

Video 1. Set up the Swim Tunnel Respirometer

2. Replace the dissolved oxygen densitometer, which is inserted into the inner swimming chamber, with fluorescent laser pointer for visualization of fluorescent beads (Video 2).

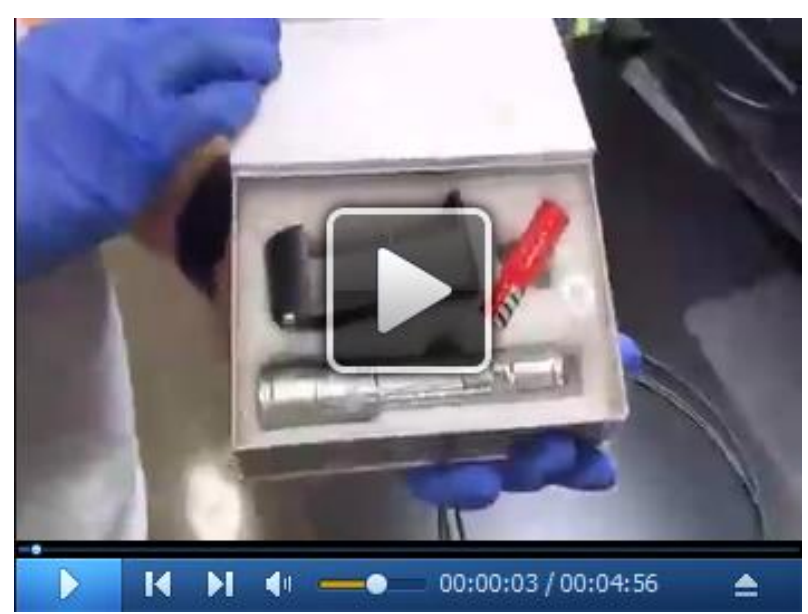

Video 2. Calibration of water velocity

3. Suspend $1 \mathrm{mg}$ of fluorescent beads in $2 \mathrm{ml}$ of water and then apply it to the double cylinder through a water inlet at the top of the side cover of the double cylinder.

4. Start the AutoResp software and set the input voltage at $0.5 \mathrm{~V}$, initiating rotation of the propeller to generate water flow in the inner swimming chamber (Figure 1C) (https://loligosystems.com/FileCatalog/GetCatalogltemFileUpload?catalogltemld=82).

5. Start the uEye Cockpit software for video capturing and record the movement of fluorescent beads in the inner swimming chamber (https://en.ids-imaging.com/ueye-cockpit.html).

6. Repeat Steps $\mathrm{A} 4$ and $\mathrm{A} 5$ by changing input voltage from 0.5 to $4.5 \mathrm{~V}$ in a stepwise manner with a $0.25 \mathrm{~V}$ increment.

7. Analyze the speed of $\sim 10,000$ fluorescent beads in movies using the DPTV software and calculate the water velocity at each input voltage. The user manual of the DPTV software is available here. 
8. Make a graph of input voltage and water velocity with a best-fit approximate line using Microsoft Excel (Figure 2).

9. Input the graph parameters in the AutoResp software so as to automatically calibrate the input voltage and water velocity.

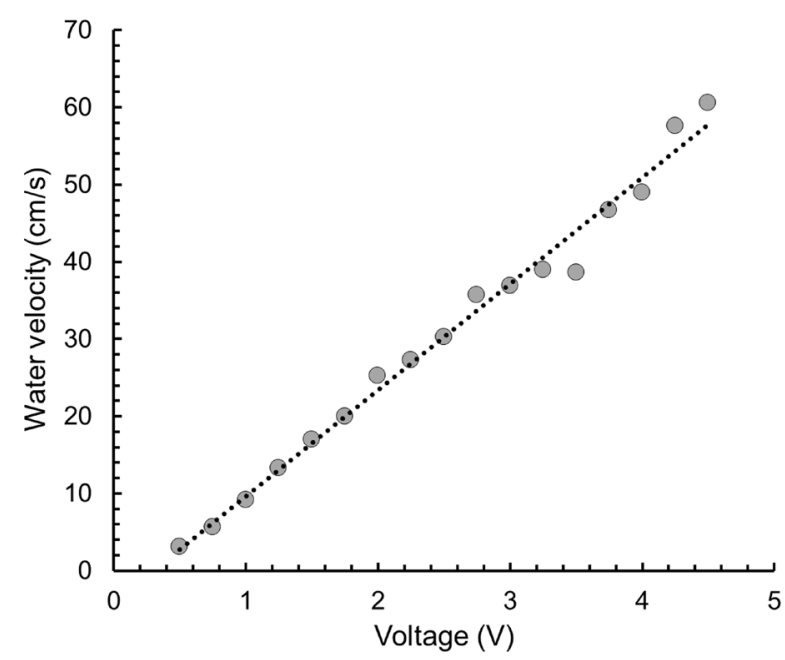

Figure 2. Voltage-Water velocity plot. The approximate line becomes straight because water velocity is proportional to the input voltage.

B. Calibration of dissolved oxygen concentration

The probe of dissolved oxygen densitometer, which can be inserted into the inner swimming chamber during swimmill assay, records the dissolved oxygen (\%) per saturated dissolvable oxygen (constant) at the water temperature. This calibration of dissolved oxygen densitometer can be carried out in the AutoResp software using oxygen-saturated water $(100 \%)$ and sodium sulfite solution $(0 \%)$.

1. Place the probe about $5 \mathrm{~cm}$ depth in $40 \mathrm{ml}$ of water in a $50 \mathrm{ml}$ conical tubes and check the dissolved oxygen in the AutoResp software (Video 3).

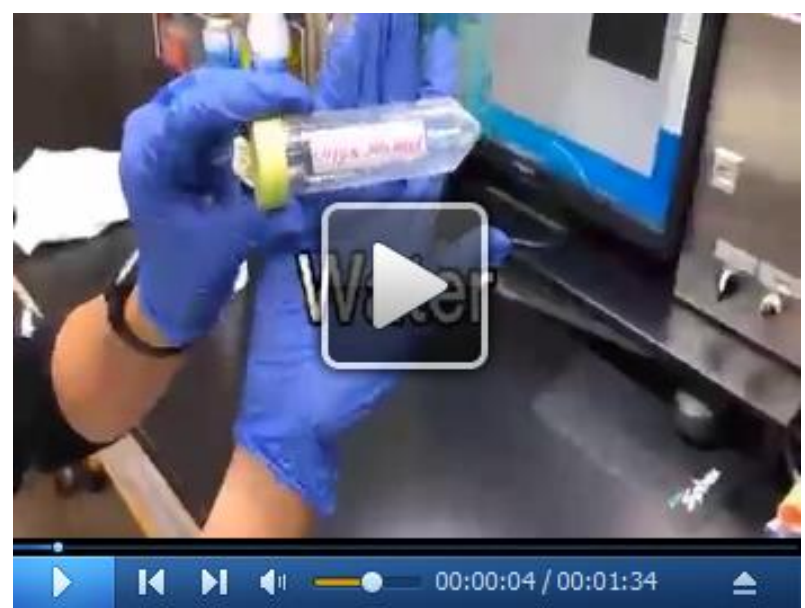

Video 3. Calibration of dissolved oxygen concentration 
2. Bubble $\sim 5 \mathrm{~L}$ of oxygen from an oxygen can into water through a nozzle (inner diameter $1 \mathrm{~mm}$ ) until the value of dissolved oxygen no longer increase.

3. Calibrate the probe for $100 \%$ by setting "Lock Hi" in the AutoResp software.

4. Dissolve $2 \mathrm{~g}$ of sodium sulfite, which is an oxygen scavenger agent, in $40 \mathrm{ml}$ of water to make oxygen-free water.

5. Clean the probe and place it in the oxygen-free water then calibrate the probe for $0 \%$ by setting "Lock Lo" in the AutoResp software.

C. Measurement of swimming speed

1. Zebrafish (Danio rerio) are reared and maintained at $26-28{ }^{\circ} \mathrm{C}$ under a $14 \mathrm{~h}$ light and $10 \mathrm{~h}$ dark photoperiod and fed twice a day in the regular care. Zebrafish used for swimming assay are kept unfed for 20 24 $\mathrm{h}$ prior to the measurement, because swimming speed of zebrafish decreases after eating food. This measurement is done between AM 10:00 and PM 3:00.

2. Put an adult zebrafish into a $10 \mathrm{ml}$ measurement cylinder $(\Phi 11 \mathrm{~mm})$ that contains $5 \mathrm{ml}$ of water and measure the volume (increase of water height) and the mass of the fish by an electric balance (Figure 3).

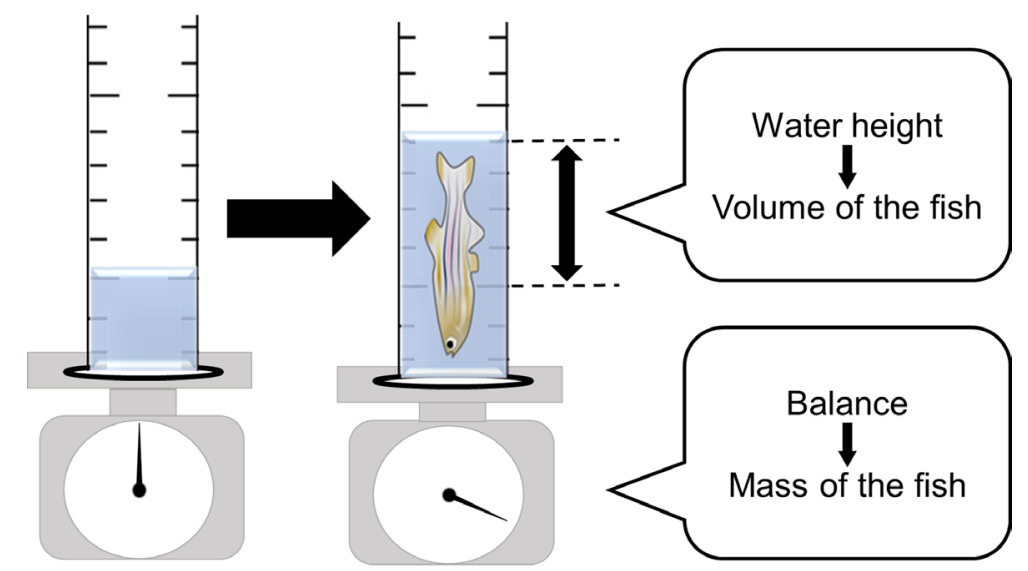

Figure 3. Measurement of the volume and mass of adult fish. The increment of the water height by putting a zebrafish in the measurement cylinder is the volume of the fish. The weight of the fish is simultaneously measurable using an electric balance.

3. Transfer the zebrafish into a $50 \mathrm{ml}$ plastic tube filled with water and put it in the outside tank for $10 \mathrm{~min}$ to acclimate the fish to the narrow cylinder and water temperature $\left(26 \pm 0.2^{\circ} \mathrm{C}\right)$.

4. Pre-mark $3 \mathrm{~cm}$ distance lines on the inner swimming chamber using an oil-based marker. This helps to measure total length and standard length of fish in the analysis of swimming movies (see Data analysis B).

5. Add a single zebrafish in the inner swimming chamber of the double cylinder (Video 4). 
Please cite this article as: Wakamatsu et. al., (2020). A Reproducible Protocol to Measure the Critical Swimming Speed of Adult Zebrafish,Bio-protocol 10 (16): e3712. DOI: 10.21769/BioProtoc.3712.

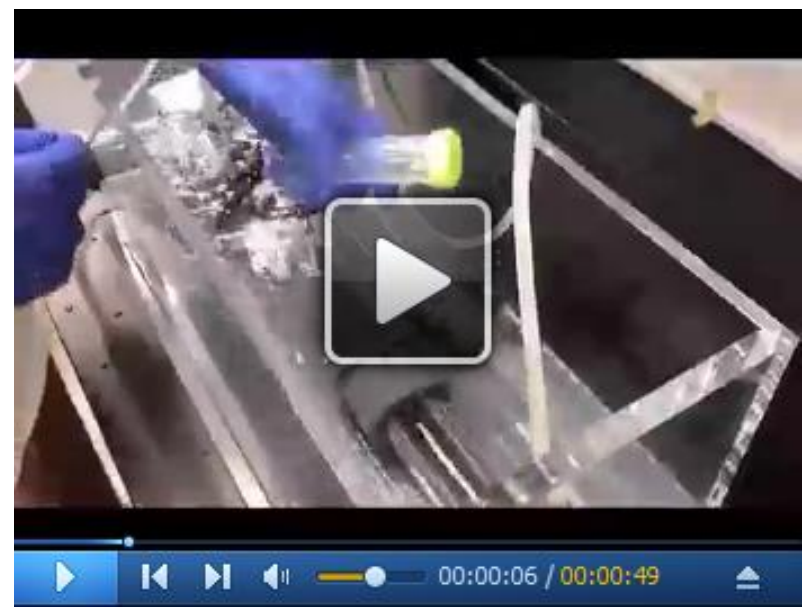

\section{Video 4. Loading zebrafish into Swim Tunnel Respirometer}

6. Set the double cylinder in the Swim Tunnel Respirometer.

7. Start the AutoResp and uEye Cockpit software to be ready for the control of input voltage and video-recording, respectively.

8. Initiate propeller rotation at $10 \mathrm{~cm} / \mathrm{s}$ water flow and video-recording simultaneously (Video 5).

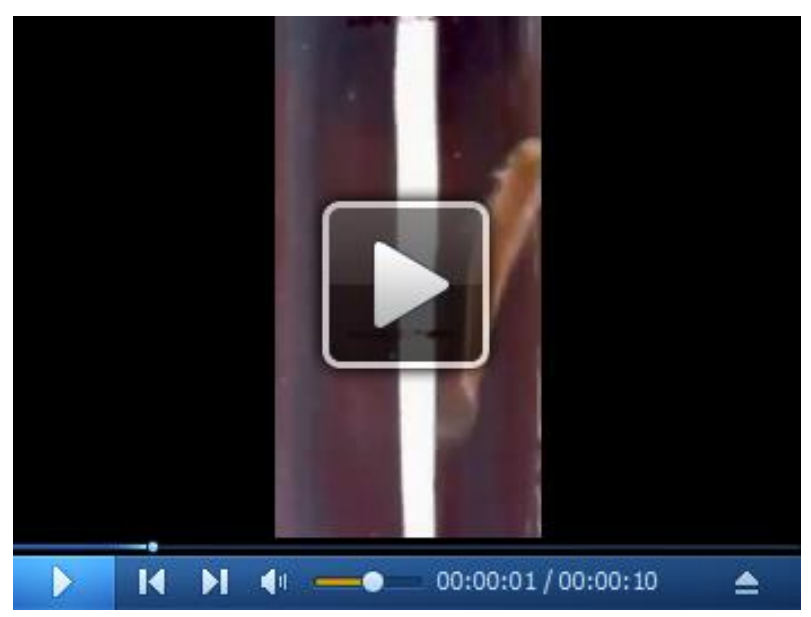

Video 5 . A zebrafish could keep swimming in $10 \mathrm{~cm} / \mathrm{s}$ water velocity

9. After $1 \mathrm{~min}$ of warming-up at $10 \mathrm{~cm} / \mathrm{s}$ water flow, the water velocity is set at $15 \mathrm{~cm} / \mathrm{s}$ to start automatic step protocol in the AutoResp software (Figure 4). 


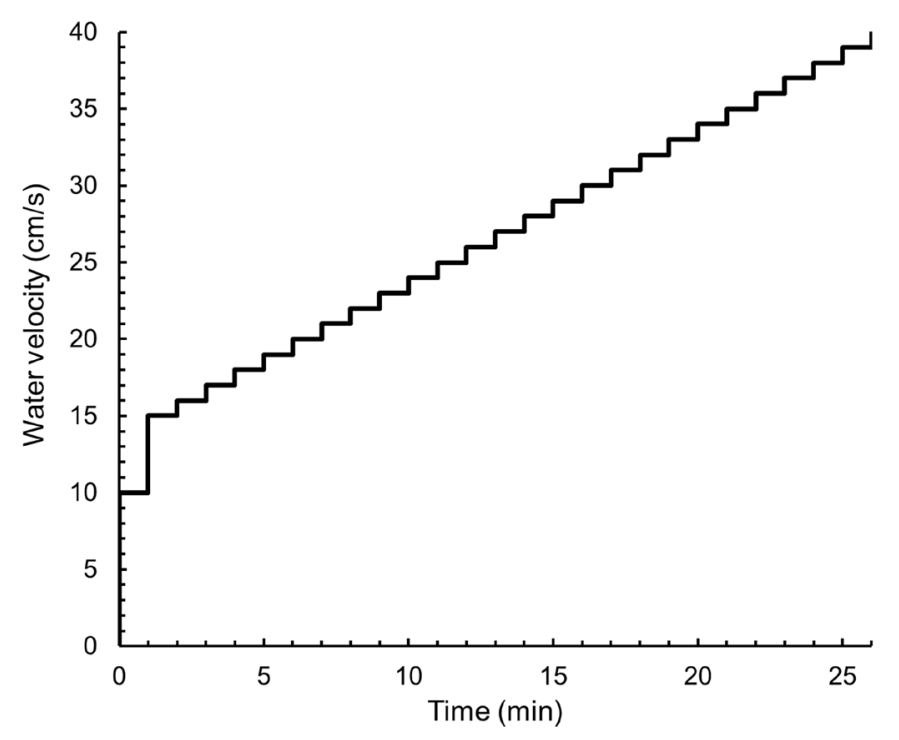

Figure 4. A protocol of the swimming time and water velocity. The water velocity is initially set at $10 \mathrm{~cm} / \mathrm{s}$ for $1 \mathrm{~min}$ and eventually increased to $15 \mathrm{~cm} / \mathrm{s}$ for $1 \mathrm{~min}$. Thereafter step protocol increases the water velocity $1 \mathrm{~cm} / \mathrm{s}$ every $1 \mathrm{~min}$.

10. After the fish get fatigued and failed to continue swimming (Video 6), stop the propeller rotation and video-recording.

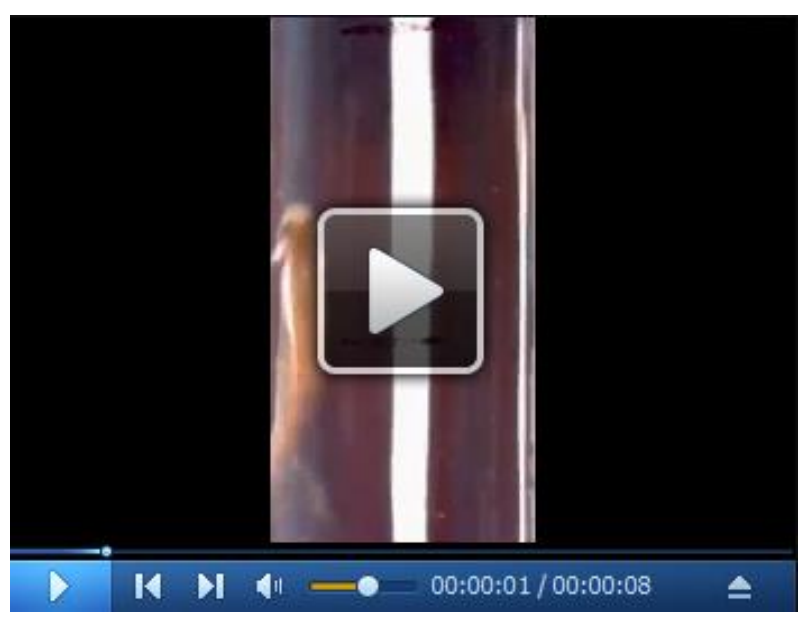

Video 6. A zebrafish failed to keep swimming in $33 \mathrm{~cm} / \mathrm{s}$ water velocity

11. Record the water temperature and dissolved oxygen concentration as well as water velocity and time when the fish failed to keep swimming.

Note: If you want to change the water temperature, you can increase the temperature by altering the setting of thermal heater or decrease it by putting an ice bag in the outside water tank. 


\section{Data analysis}

A. Calculation of the critical swimming speed

The critical swimming speed $\left(U_{\text {crit }}\right)$ is the maximum water velocity in which fish can keep swimming. The $U_{\text {crit }}$ of fish is calculated according to the established formula (Brett, 1964). $U_{\text {crit }}=U_{\max }+T / 60$. $U_{\max }(\mathrm{cm} / \mathrm{s})$ : the highest water velocity when zebrafish continued to swim for whole $1 \mathrm{~min}$. $T(\mathrm{~s})$ : time elapsed when fish failed to keep swimming in $1 \mathrm{~min}$.

B. Measurement of the body size

Three different definitions of body size have been established; total length, standard length and caudal fin length (Figure 5) (Plaut, 2000). The total length and standard length are measurable in comparison with pre-marked $3 \mathrm{~cm}$ distance lines in a frame of swimming movies (dorsal view). In ImageJ software, draw a line of $3 \mathrm{~cm}$ distance and measure the length by pixel using the Analyze/Measure function. Similarly, draw a line along the midline of the fish from the head to the tail and measure the length by pixel and then calculate the actual length by hands. The standard length is calculated by the line from the head to the root of caudal fin. The difference of total and standard lengths is the caudal fin length. The motor ability of zebrafish varies by the length of caudal fin (Wakamatsu et al., 2019).

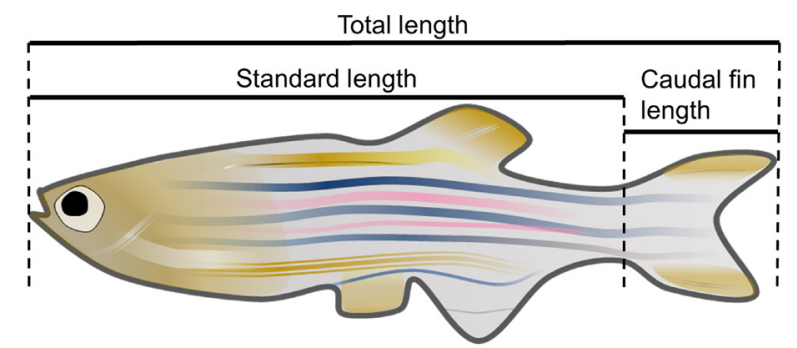

Figure 5. The definition of body size of zebrafish. Three different parameters of body size are established in fish studies.

\section{Notes}

We could obtain reproducible results using this protocol (Wakamatsu et al., 2019).

\section{Acknowledgments}

We thank Hirata Lab members for helpful comments. This work was supported by a Grant-in-Aid for Scientific Research B (16H04657, 19H03329) and Scientific Research on Innovative Areas (17H05578) from the MEXT, Japan, the Naito Foundation and the Japan Epilepsy Research Foundation. 
Please cite this article as: Wakamatsu et. al., (2020). A Reproducible Protocol to Measure the Critical Swimming Speed of Adult Zebrafish,Bio-protocol 10 (16): e3712. DOI: 10.21769/BioProtoc.3712.

\section{Competing interests}

The authors declare no competing interests.

\section{Ethics}

All animal experiments described in this manuscript and guidelines for use of zebrafish have been approved by Animal Care and Use Committee at Aoyama Gakuin University (A9: valid until March 2021).

\section{References}

1. Brett, J. (1964). The respiratory metabolism and swimming performance of young sockeye salmon. J Fish Research Board of Can 21: 1183-1226.

2. Conradsen, C. and McGuigan, K. (2015). Sexually dimorphic morphology and swimming performance relationships in wild-type zebrafish Danio rerio. J Fish Biol 87(5): 1219-1233.

3. Conradsen, C., Walker, J. A., Perna, C. and McGuigan, K. (2016). Repeatability of locomotor performance and morphology-locomotor performance relationships. J Exp Biol 219(Pt 18): 2888-2897.

4. Gemberling, M., Karra, R., Dickson, A. L. and Poss, K. D. (2015). Nrg1 is an injury-induced cardiomyocyte mitogen for the endogenous heart regeneration program in zebrafish. Elife 4: e05871.

5. Gilbert, M. J. H., Zerulla, T. C. and Tierney, K. B. (2014). Zebrafish (Danio rerio) as a model for the study of aging and exercise: physical ability and trainability decrease with age. Experimental Gerontology 50: 106-113.

6. Leris, I., Sfakianakis, D. G. and Kentouri, M. (2013). Are zebrafish Danio rerio males better swimmers than females? J Fish Biol 83(5): 1381-1386.

7. Lucas, J., Percelay, I., Larcher, T. and Lefrancois, C. (2016). Effects of pyrolytic and petrogenic polycyclic aromatic hydrocarbons on swimming and metabolic performance of zebrafish contaminated by ingestion. Ecotoxicol Environ Saf 132: 145-152.

8. Massé, A. J., Thomas, J. K. and Janz, D. M. (2013). Reduced swim performance and aerobic capacity in adult zebrafish exposed to waterborne selenite. Comp Biochem Physiol C Toxicol Pharmacol 157(3): 266-271.

9. Messerli, M., Aaldijk, D., Haberthur, D., Ross, H., Garcia-Poyatos, C., Sande-Melon, M., Khoma, O. Z., Wieland, F. A. M., Fark, S. and Djonov, V. (2020). Adaptation mechanism of the adult zebrafish respiratory organ to endurance training. PLoS One 15(2): e0228333.

10. Mokalled, M. H., Patra, C., Dickson, A. L., Endo, T., Stainier, D. Y. and Poss, K. D. (2016). Injury-induced ctgfa directs glial bridging and spinal cord regeneration in zebrafish. Science 354(6312): 630-634. 
Please cite this article as: Wakamatsu et. al., (2020). A Reproducible Protocol to Measure the Critical Swimming Speed of Adult Zebrafish,Bio-protocol 10 (16): e3712. DOI: 10.21769/BioProtoc.3712.

11. Palstra, A. P., Tudorache, C., Rovira, M., Brittijn, S. A., Burgerhout, E., van den Thillart, G. E., Spaink, H. P. and Planas, J. V. (2010). Establishing zebrafish as a novel exercise model: swimming economy, swimming-enhanced growth and muscle growth marker gene expression. PLoS One 5(12): e14483.

12. Parisi, A., Blattmann, P., Lizzo, G., Stutz, V., Strohm, L., Richard, J., Civiletto, G., Charpagne, A., Raymond, F., Gobet, C., et al. (2018). PGC1a and Exercise Adaptations in Zebrafish. BioRxiv 483784.

13. Plaut, I. (2000). Effects of fin size on swimming performance, swimming behaviour and routine activity of zebrafish Danio rerio. J Exp Biol 203(Pt 4): 813-820.

14. Thomas, J. K., Wiseman, S., Giesy, J. P. and Janz, D. M. (2013). Effects of chronic dietary selenomethionine exposure on repeat swimming performance, aerobic metabolism and methionine catabolism in adult zebrafish (Danio rerio). Aquatic Toxicology 130: 112-122.

15. Wakamatsu, Y., Ogino, K. and Hirata, H. (2019). Swimming capability of zebrafish is governed by water temperature, caudal fin length and genetic background. Sci Rep 9(1): 16307.

16. Widrick, J. J., Gibbs, D. E., Sanchez, B., Gupta, V. A., Pakula, A., Lawrence, C., Beggs, A. H. and Kunkel, L. M. (2018). An open source microcontroller based flume for evaluating swimming performance of larval, juvenile, and adult zebrafish. PLoS One 13(6): e0199712. 